codes encompassing the materials, skills and meanings that constitute online partner-seeking as a social practice.

Findings Online partner seeking is a social practice normalised in contemporary culture, enmeshed within broader online cultures of image presentation. It is associated with multiple goals and imbued with possibilities as well as risks. Material elements we identified related to the technology, its affordances, and how these shape interactions. We found that technological, interpersonal, and self-care skills were together required to seek and progress to various relationship forms and protect the self. Distinct linguistic, sexual, harm/damage limitation and exit strategies also drew on a range of skills. Participants reflected on how they presented themselves online, on their intentions, and on the skills required to 'read' situations and act authentically.

Conclusion While online partner seeking has often been considered individualistic, outcomes can be read through a lens of Social Practice Theory. Successful partner selection, communication and avoidance of harm depend on a complex learned constellation of the skills, materials and meanings associated with dating choices. Our findings have potential to inform survey questionnaire design and effective, nuanced health promotion interventions which consider intersecting dimensions of this social practice to build skills, develop goals and assess strategies to respond to unwanted interaction.

\section{P258 EXPLORING THE ATTITUDES AND ACCEPTABILITY OF ANAL SELF-EXAMINATION FOR EARLY DETECTION OF ANAL SYPHILIS - A MIXED METHOD STUDY}

${ }^{1} \mathrm{E}$ Aung ${ }^{*},{ }^{1,2} \mathrm{~J}$ Ong, ${ }^{1,2} \mathrm{~T}$ Phillips, ${ }^{1,2} \mathrm{M}$ Chen, ${ }^{1,2} \mathrm{~K}$ Maddaford, ${ }^{1,2} \mathrm{E}$ Rodriguez, ${ }^{1,2} \mathrm{C}$ Fairley, 1,2,3E Chow. ${ }^{1}$ Melbourne Sexual Health Centre, Carlton, Australia; ${ }^{2}$ Central Clinical School, Faculty of Medicine, Nursing and Health Sciences, Monash University, Melbourne, Australia; ${ }^{3}$ Centre for Epidemiology and Biostatistics, Melbourne School of Population and Global Health, The University of Melbourne, Melbourne, Australia

10.1136/sextrans-2021-sti.333

Objective Studies have suggested MSM practising receptive anal sex are more likely to miss anorectal syphilis lesions. We hypothesised that performing regular anal self-examination (ASE) could detect anorectal syphilis lesions. We aimed to explore the attitudes and acceptability of MSM on performing ASE to detect primary anal syphilis.

Methods This mixed-method study involved semi-structured interviews with 20 MSM purposively sampled from a sexual health clinic and a cross sectional survey with MSM over 18 years of age recruited through the clinic and social media between January to November 2020. Interviews data were analysed thematically and descriptive analysis performed on the survey data.

Results Four major themes emerged from the interviews; reasons for ASE, preferred resources for ASE, acceptability of ASE, and attitudes towards partner anal examination. The majority of MSM had conducted ASE previously; however, only a few performed it regularly for medical reasons. Most men not regularly conducting ASE were uncertain about differentiating normal and abnormal ASE findings, although were willing to perform ASE regularly with appropriate education and training.
574 MSM completed the survey (median age: 34 [IQR 2745]): 182 (32\%) had previously performed ASE. Among 373 (65\%) who had not performed ASE, 250 (67\%) were willing to consider ASE as a regular practice for early syphilis detection (median 0.9 times per month). There were no significant differences in demographic characteristics and sexual practices between the two groups.

Conclusion Most MSM were willing to perform ASE, however, would like further education and training to gain more confidence in performing ASE as a screening tool. Almost two-third of the MSM who had never performed ASE were willing to adopt ASE practice in the future. Further studies are required to explore the adherence and cost-effectiveness of ASE.

\section{P259 SEX IN THE CITY: UNDERSTANDING PERCEIVED RISKS AND SEXUAL BEHAVIOR AMONG ADOLESCENTS AND YOUNG ADULTS DURING THE SARS CO-V-2 PANDEMIC}

M Trent*, A Agwu, J Perin, J Rowell, L Spatafore, J Coleman, C Gaydos, P Matson. Johns Hopkins School of Medicine, Baltimore, USA

\subsection{6/sextrans-2021-sti.334}

Background SARS Co-V-2 (COVID-19) mitigation strategies have resulted in limited clinical operations for sexual health services and adolescents and young adults (AYA) have received mixed messages about the risk for serious COVID-19 infection. This work describes the sexual behavior and relationship between COVID-19 risk perceptions and sexual behaviors among urban AYA during the pandemic.

Methods Cross-sectional data were used from AYA enrolled across four sexual health studies in Baltimore, Maryland (USA). Participants had an active STI, HIV, or were at risk for STI/HIV. The March 16, 2020 'stay-at-home' orders were used to define the pandemic period in the human subjects' approved telephone survey. Demographics, COVID-19 risk perception, testing behavior, positivity in their social circle, and relationship/sexual behaviors data were evaluated using regression analyses.

Results 194 participants with a mean (sd) age of 22.7 (2.8) years were surveyed. Most were female (81\%) with public health insurance $(68 \%), 61 \%$ were in a relationship, $36 \%$ were cohabiting, $79 \%$ had sex during the stay-at-home order, $68 \%$ used some form of contraception, 39\% used a condom at last sex, and $23 \%$ had STI screening during the pandemic. 51\% had COVID-19 testing, and $31 \%$ and $8 \%$ experienced a COVID-19-related diagnosis or death, respectively, in their social circle. Using a 10-point Likert scale $(10=$ most concerned), the mean concern score for contracting COVID-19 was 5.0 (3.7) and during intercourse was 3.0 (4.1). Those with COVID-19 positivity within their social circle were marginally more likely to have COVID-19 testing (adjusted OR 1.69, 95\% CI $0.89-3.19, \mathrm{p}=$ 0.107). Concern for COVID infection or COVID-19 in social circle was not associated with sexual intercourse or condom use.

Conclusion Urban AYAs remain at risk for STIs and COVID19 given community rates, low condom use, and low COVID risk perceptions. Youth-focused mitigation strategies to reduce STI/HIV and COVID-19 are warranted. 